IC-S2-02 TRACKING DISEASE PROGRESSION WITH VOLUMETRIC MRI

Nick Fox, Institute of Neurology, University College London, London, United Kingdom.Contacte-mail: cdecarli@ucdavis.edu

Abstract not available.

IC-S2-03 INTEGRATING MR TECHNIQUES TO STUDY ALZHEIMER'S DISEASE

Frederik Barkhof, Alzheimer Center, VU Medical Center, Amsterdam, Netherlands.Contacte-mail: cdecarli@ucdavis.edu

Abstract not available.

SATURDAY, JULY 11, 2009
ALZHEIMER'S IMAGING CONSORTIUM PRESENTATIONS
ORAL
IC-O1

IC-O1-01 GENETIC PREDICTORS OF 12-MONTH CHANGE IN MRI HIPPOCAMPAL VOLUME IN THE ALZHEIMER'S DISEASE NEUROIMAGING INITIATIVE COHORT: ANALYSIS OF LEADING CANDIDATES FROM THE ALZGENE DATABASE

Andrew J. Saykin, Li Shen, Shannon L. Risacher, Sungeun Kim, Kwangsik Nho, John D. West, Tatiana M. Foroud, and the Alzheimer's Disease Neuroimaging Initiative, Indiana University School of Medicine, Indianapolis, IN, USA. Contact e-mail: asaykin@iupui.edu

Background: High density genome-wide microarray data and one year longitudinal MRI data have become available for most participants of the Alzheimer's Disease Neuroimaging Initiative (ADNI), a multi-center longitudinal study assessing imaging in the diagnosis and longitudinal monitoring of Alzheimer's disease (AD) and amnestic mild cognitive impairment (MCI). The AlzGene database provides meta-analytic data on leading candidate genes for AD. Here, we analyzed whether common variants in current major candidate genes could predict longitudinal changes on MRI. Methods: Baseline and 12 month T1-weighted MP-RAGE scans acquired on $1.5 \mathrm{~T}$ magnets from 627 ADNI participants (141 AD, 60 MCI-Converters, 241 MCI-Stable, 185 healthy controls, HC) were analyzed using Freesurfer software for automated parcellation and SPM5 for voxel-based morphometry (VBM). Genetic data consisted of APOE alleles and the Illumina 610 Quad array that includes over 620,000 features. For this analysis, common (minor allele frequency, MAF > 0.2) single nucleotide polymorphisms (SNPs) from the top 30 candidate genes from the AlzGene database were examined. Regression models were performed using SAS/Stat 9.3 to test the ability of SNPs to predict hippocampal volume and gray matter density changes. Results: Models including diagnosis group (AD, MCI-C, MCI-S, HC), SNP $(0,1,2)$, parental history of dementia $(0,1,2)$, APOE epsilon 4 status $(0,1)$ and all interactions were computed. 732 SNPs were available for analysis with MAF $>0.2$. A threshold of $p$ $<0.00001$ was employed to reduce the likelihood of false discoveries. Using this model and criterion, 5 genes showed significant SNPs associated with hippocampal volume changes (NEDD9, SORL1, DAPK1, IL1B, SORCS1). In addition, SNPs from several other candidates genes showed less robust indications of possible association (at $\mathrm{p}<.0001$ : MYH13, TNK1; at $\mathrm{p}<.001$ : ACE, PRNP, MAPT, PCK1, GAPDHS and APP). Conclusions: Variation in major candidate genes was significantly related to 12 month longitudinal change in hippocampal volume in the ADNI cohort. These findings suggest that combining genetics and imaging with other clinical data may yield refined prediction models for those at highest risk of progression. Replication and further investigation are warranted. As putatively disease modifying agents are developed, the ability to determine those at highest risk for progression will be critical.

\section{IC-O1-02 NEURAL UNDERPINNINGS OF AUTOBIOGRAPHICAL MEMORY RETRIEVAL IN ALZHEIMER'S DISEASE}

Olga Meulenbroek, Roy P. C. Kessels, Marcel G. M. Olde Rikkert,

Mark Rijpkema, Guillén Fernández, Donders Institute for Brain, Cognition and Behaviour, Nijmegen, Netherlands.Contacte-mail: o.meulenbroek@ fcdonders.ru.nl

Background: Patients with Alzheimer's Disease (AD) show remarkably intact very-long-term memory. However, they seem to base their remote memories on factual knowledge (i.e. semantic memory) and less on an actual re-experience of the episode. This shift is probably related to structural decline of the hippocampi, and leads to the hypothesis that $\mathrm{AD}$ patients will attempt to compensate by activating for instance the left inferior frontal gyrus (LIFG; semantic processing) and the ventromedial prefrontal cortex (vmPFC; interacts with hippocampus during consolidation). Methods: Using fMRI, we investigated 22 healthy elderly control subjects (aged 69.6 \pm 8.6 , MMSE $29.0 \pm 1.1$ ) and 21 patients with early stage probable AD (aged 72.4 \pm 7.1 , MMSE 25.1 \pm 3.2 ), according to the research criteria by Dubois (Lancet Neurology 2007). Matched Autobiographical and Semantic (world knowledge) statements (true:false $=3: 1$ ) were visually presented to the subjects, who indicated if the statements were true (button press). Autobiographical statements were derived from an interview occurring $>6$ weeks before scanning. Memories were transcribed and scored, according to the Autobiographical Interview Scoring Manual (Levine et al, Psychology and Aging 2002). Results: While reported memories were of similar length, AD patients reported less episodic details $\left(F_{(1,41)}\right.$ $=17.70, \mathrm{P}<.001)$, and more semantic details than healthy elderly $\left(\mathrm{F}_{(1,41)}=13.15, \mathrm{P}<.005\right)$. During fMRI, performance on the Autobiographical $\left(\mathrm{F}_{(1,41)}=15.89, \mathrm{P}<0.001\right)$ and the Semantic $\left(\mathrm{F}_{(1,41)}=\right.$ 21.88, $\mathrm{P}<0.001)$ condition was lower for the AD patients. All subjects activate a network involved in autobiographical retrieval during fMRI, when we contrast autobiographical versus semantic memory (A $>$ S). Controlling the data for performance, $\mathrm{AD}$ patients showed enhanced activity in LIFG, vmPFC, right precuneus and left lingual gyrus compared to healthy elderly. Also, AD patients had smaller hippocampi $\left(\mathrm{t}_{40}=2.02, \mathrm{P}<0.05\right)$. Moreover, activation of LIFG and vmPFC was significantly negatively correlated with hippocampal volume in the AD patients only $(r=-0.40$, $\mathrm{P}<0.05$ and $\mathrm{r}=-0.37, \mathrm{P}<0.05)$, indicating the linking function of the hippocampus is moved to the vmPFC (like in consolidation), and patients rely more on semantic processing. Conclusions: AD patients attempt to compensate for their hippocampal damage/ autobiographical memory impairment by relying more on the vmPFC as a linking node and by increasing semantic processing.

\section{IC-O1-03 FROM MILD COGNITIVE IMPAIRMENT TO ALZHEIMER'S DISEASE: COMPARISONS OF METABOLIC AND STRUCTURAL BRAIN ALTERATION EVOLUTIONS}

Nicolas Villain ${ }^{1}$, Marine Fouquet ${ }^{1}$, Brigitte Landeau ${ }^{1}$, Mezenge Florence ${ }^{1}$, Vincent de la Sayette ${ }^{1,2}$, Fausto Viader ${ }^{1,2}$, Jean-Claude Baron ${ }^{3}$,

Francis Eustache ${ }^{1}$, Béatrice Desgranges ${ }^{1}$, Gaël Chételat ${ }^{1},{ }^{1}$ Inserm- $E$ PHE-Université de Caen Basse-Normandie U923, Caen, France; ${ }^{2}$ Département de Neurologie, CHU Côte de Nacre, Caen, France $;{ }^{3}$ Department of Clinical Neurosciences, Neurology Unit, University of Cambridge, Cambridge, United Kingdom.Contacte-mail: villain@cyceron.fr

Background: Several studies have highlighted the evolution of functional or structural brain alterations from amnestic mild cognitive impairment (aMCI) to Alzheimer's disease (AD). They have pointed to a progression of brain atrophy within temporal, parietal and frontal lobes ${ }^{1,2}$ while metabolic decreases were restricted to frontal and cingulate areas ${ }^{3,4}$. Crosssectional studies in $\mathrm{AD}$ led to a similar discrepancy between structural and functional alterations ${ }^{5}$. This study aims at directly assessing the relationships and differences between the profiles of metabolic and morphological changes from aMCI to AD. Methods: Seventeen aMCI patients underwent both ${ }^{18}$ FDG-PET and T1-MRI examinations at inclusion (t0) and 18 months later (t18). At t18, seven of them fulfilled clinical criteria for probable $\mathrm{AD}$ (converters) while the others remained clinically stable (non-converters). For MRI data, we used VBM5.1 for segmentation and DARTEL $^{6}$ for a two-step warping procedure (first to an intermediate 't0-t18' subject template and second to a mean group template). The t0 